\title{
Percutaneous Pedicle Cannulation: An in-vitro Study Assessing Clinical Expertise versus Technology
}

\author{
Y. Raja Rampersaud and Henry Ahn
}

\begin{abstract}
Division of Orthopaedic and Neurosurgery, Univ. of Toronto. Toronto Western Hospital, University Health Network, ECW-1-039, 399 Bathurst St., Toronto, Ontario, M5T-2S8, Canada.
\end{abstract}

\begin{abstract}
Percutaneous procedures represent an opportunity to evaluate the role of technology (e.g. imaging or computer assisted surgery) in enhancing versus replacing clinical expertise (both procedural and appropriate application of a given technology). The objective of this study was to assess the role of clinical experience in the accuracy of percutaneous pedicle cannulation using virtual fluoroscopy or conventional $\mathrm{C}$-arm fluoroscopy. There were significantly $(\mathrm{p}=0.004)$ more pedicle breaches for the resident $(8 / 30)$ compared to the experienced spine surgeon (1/30). the result of this study supports the importance of clinical expertise for the performance of technically demanding procedures with or without new technologies.
\end{abstract}

\section{Objective}

The objective of this study was to assess the role of clinical experience in the accuracy of percutaneous pedicle cannulation using virtual fluoroscopy or conventional C-arm fluoroscopy.

\section{Background}

Recent procedures such as vertebroplasty, kyphoplasty and minimally invasive pedicle screw instrumentation require percutaneous cannulation of spinal pedicles. The technical challenges and misplacement rates of open pedicle screw placement have been well documented in the literature. In contrast, these issues have not been well addressed for percutaneous pedicle cannulation.

\section{Methods}

Using an 11-gauge bone biopsy needle, sixty pedicles (2 cadavers, bilateral, T4- S1) were cannulated using virtual fluoroscopy (VF) or conventional C-arm fluoroscopy (CF). An experienced spinal surgeon performed half of the procedures and a $3^{\text {rd }}$ year orthopaedic resident performed the other half. To eliminate learning from one level to the next, the procedures were randomized to technique (VF or CF), level, side (right or left) and surgeon (resident or spine surgeon). Post-procedure, the cadavers underwent fine cut computed tomography using a 3D image-guidance scanning 
protocol. The positions of the needles were graded using a point system as follows: A-location relative to the pedicle (1-completely within the pedicle, 2-pedicle wall breach $<2 \mathrm{~mm}$, 3-pedicle wall breach 2-4 $\mathrm{mm}$, 4-pedicle wall breach $>4 \mathrm{~mm}$ ); Bdirection of pedicle breech if one occurred (1-surperior, 2-lateral, 3-inferior, 4medial); C-tube trajectory (1-ideal, 2-straight, 3-lateral, 4-medial) and D-tube position within the vertebral body (1-in, 2-out). A cumulative point score was tabulated for each pedicle cannulation.

\section{Results}

There were significantly $(p=0.004)$ more pedicle breaches for the resident $(8 / 30)$ compared to the experienced spine surgeon (1/30). There was no difference in pedicle breaches comparing the $\mathrm{VF}$ to $\mathrm{CF}$ techniques for the resident (VF-4 and $\mathrm{CF}$ 4breaches) or spinal surgeon (VF-0 and CF-1 breach). All breaches were in the thoracic spine as follows: Resident (VF) - T4 (A-3, B-4, C-4, D-1), T6 (A-3, B-2, C3, D-2), T9 (A-2, B-2, C-1, D-1), T11 (A-3, B-2, C-3, D-2); Resident (CF) - T4 (A-3, B-4, C-4, D-1), T6 (A-3, B-2, C-3, D-2), T8 (A-2, B-2, C-1, D-1), T9 (A-2, B-3, C-1, D-1); Spine surgeon (VF) - T7 (A-2, B-3, C-1, D-1).

\section{Discussion}

Percutaneous pedicle screw cannulation represents an increased degree of technical difficulty compared to the open procedure. The loss of visual anatomic landmarks mandates the use of imaging and challenges the limits of clinical expertise (anatomical knowledge, procedural skill, and clinical experience). Consequently, percutaneous procedures represent an opportunity to evaluate the role of technology (e.g. imaging or computer assisted surgery) in enhancing versus replacing clinical expertise (both procedural and appropriate application of a given technology). In this study, it is likely that the inexperienced user more often made errors such as image misinterpretation or virtual image relative to tool shifting secondary to a slightly flexible probe or intersegmental motion. Therefore, similar types or numbers of errors can occur with either lack of clinical expertise for a given procedure or the application of technology that procedure. Rigorous training is required to gain clinical expertise for any procedure, however emerging technologies are often utilized with minimal training. As shown in this study current technologies cannot completely replace clinical expertise. It is the responsibility of both the user and the manufacturers of these technologies to ensure appropriate training and hence use of a given technology.

Percutaneous spinal procedures represent a new challenge to the CAS community. Emerging technologies continue to reduce error within CAS systems and address these challenges, however these new technologies need to be evaluated not only by experts, but also by the novice. It is the novice user who will truly bring forth end user and application error. In conclusion the result of this study supports the importance of clinical expertise for the performance of technically demanding procedures with or without new technologies. 